\title{
Current Status and Research into Overcoming Limitations of Capsule Endoscopy
}

\author{
Won Gun Kwack and Yun Jeong Lim \\ Department of Internal Medicine, Dongguk University Ilsan Hospital, Dongguk University College of Medicine, Goyang, Korea
}

Endoscopic investigation has a critical role in the diagnosis and treatment of gastrointestinal (GI) diseases. Since 2001, capsule endoscopy (CE) has been available for small-bowel exploration and is under continuous development. During the past decade, CE has achieved impressive improvements in areas such as miniaturization, resolution, and battery life. As a result, CE is currently a firstline tool for the investigation of the small bowel in obscure gastrointestinal bleeding and is a useful alternative to wired enteroscopy. Nevertheless, CE still has several limitations, such as incomplete examination and limited diagnostic and therapeutic capabilities. To resolve these problems, many groups have suggested several models (e.g., controlled $\mathrm{CO}_{2}$ insufflation system, magnetic navigation system, mobile robotic platform, tagging and biopsy equipment, and targeted drug-delivery system), which are in development. In the near future, new technological advances will improve the capabilities of $\mathrm{CE}$ and broaden its spectrum of applications not only for the small bowel but also for the colon, stomach, and esophagus. The purpose of this review is to introduce the current status of CE and to review the ongoing development of solutions to address its limitations. Clin Endosc 2016;49:8-15

Key Words: Capsule endoscopy; Small bowel; Colon; Esophagus

\section{INTRODUCTION}

Capsule endoscopy (CE) has been available in clinical practice for the evaluation of small-bowel disease since 2001. CE has most commonly been used in cases of obscure gastrointestinal bleeding (OGIB). ${ }^{1}$ Several small-bowel capsules (PillCam, Given Imaging, Yoqneam, Israel; EndoCapsule, Olympus, Tokyo, Japan; MiroCam, IntroMedic, Seoul, Korea; OMOM, Jinshan Science, Chongqing, China; CapsoCam, CapsoVision, Saratoga, CA, USA) are now available worldwide. ${ }^{2} \mathrm{CE}$ has many advantages compared to conventional wired endoscopy, such as convenience and less invasiveness. However, CE still has several significant technical limitations that need to be ad-

Received: August 11, 2015 Revised: September 16, 2015

Accepted: September 16, 2015

Correspondence: Yun Jeong Lim

Division of Gastroenterology, Department of Internal Medicine, Dongguk University Ilsan Hospital, Dongguk University College of Medicine, 27 Dongguk-ro, Ilsandong-gu, Goyang 10326, Korea

Tel: +82-31-961-7133, Fax: +82-31-961-9339, E-mail: drlimyj@gmail.com

(cc) This is an Open Access article distributed under the terms of the Creative Commons Attribution Non-Commercial License (http://creativecommons.org/ licenses/by-nc/3.0) which permits unrestricted non-commercial use, distribution, and reproduction in any medium, provided the original work is properly cited. dressed technically. First, the gastrointestinal (GI) lumen is not inflated in $\mathrm{CE}$, and only passive images can be obtained while the capsule passes through the GI tract. Therefore, lesions may be missed in CE, and the ampulla of Vater or ileocecal valve could be undetected in small-bowel CE. Moreover, CE often produces obstructed images because of bile, mucus, and other factors. It takes too long to administer $\mathrm{CE}$ and interpret capsule images. Second, CE cannot be used to take a biopsy specimen, nor does it have therapeutic capabilities. To overcome these limitations, several groups have suggested alternatives, and these alternatives are currently in development. Here, we introduce results from recent research about the future of CE.

\section{CURRENT STATUS OF CAPSULE ENDOSCOPY}

\section{Small-bowel capsule endoscopy}

With the advancements in CE technology, CE has rapidly expanded the indication for investigations of the GI tract. Given Imaging has installed more than 4,250 centers in about 60 countries and has sold $>650,000$ capsules. CE is a particularly 
Table 1. Video Capsule Endoscopy

\begin{tabular}{|c|c|c|c|c|c|c|c|}
\hline \multirow{2}{*}{ Variable } & \multicolumn{3}{|c|}{ PillCam } & \multirow{2}{*}{ EndoCapsule } & \multirow{2}{*}{ MiroCam } & \multirow{2}{*}{ OMOM } & \multirow{2}{*}{ CapsoCam } \\
\hline & SB3 & ESO2 & Colon2 & & & & \\
\hline Dimensions, $\mathrm{mm}$ & $11.4 \times 26.2$ & $11 \times 26$ & $11.6 \times 31.5$ & $11 \times 26$ & $11 \times 24.5$ & $13 \times 27.9$ & $11 \times 31$ \\
\hline Frame rate, fps & $2-6$ & 18 & $4-35$ & 2 & 3 & 2 & $20 \max$ \\
\hline Operating time & $8 \mathrm{hr}$ & $30 \mathrm{~min}$ & $10 \mathrm{hr}$ & $8 \mathrm{hr}$ & $12 \mathrm{hr}$ & $6-8 \mathrm{hr}$ & $15 \mathrm{hr}$ \\
\hline Imaging heads & 1 & 2 & 2 & 1 & 1 & 1 & 4 \\
\hline Field of view, ${ }^{\circ}$ & 156 & 169 & 172 & 145 & 170 & 140 & 360 \\
\hline Transmission mode & $\mathrm{RF}$ & $\mathrm{RF}$ & $\mathrm{RF}$ & $\mathrm{RF}$ & EFP & $\mathrm{RF}$ & USB \\
\hline FDA & Yes & Yes & Yes & Yes & Yes & No & No \\
\hline Image sensor & CMOS & CMOS & CMOS & $\mathrm{CCD}$ & CMOS & $\mathrm{CCD}$ & White LEDs \\
\hline Optical enhancement & & FICE setting & & Contrast imaging & NA & NA & NA \\
\hline
\end{tabular}

fps, frames per second; RF, radiofrequency; EFP, electric field propagation; USB, universal serial bus; FDA, Food and Drug Administration; CMOS, complementary metal oxide silicon; CCD, charge-coupled device; LED, light-emitting diode; FICE, Fujinon intelligent chromoendoscopy; NA, not applicable.

useful tool for patients with suspected small-bowel disease, including OGIB, iron deficiency anemia, Crohn's disease (CD), tumors, polyposis syndromes, and celiac disease. A recent clinical guideline has recommended $\mathrm{CE}$ as a first-line investigation tool in patients with OGIB. The first available video capsule, brand name M2A (mouth to anus; Given Imaging), was approved by the Food and Drug Administration (FDA) in 2001 as an adjunctive tool for small-intestine imaging. At present, there are five small-bowel capsule endoscope models on the market worldwide. ${ }^{3,4}$ Capsule endoscope models with U.S. FDA approval consist of PillCam, EndoCapsule, and MiroCam. Although the various capsules are similar in size and shape, they differ in dimensions, frame rates, operating time, field of view, image sensor, and optical enhancements (Table 1). PillCam and MiroCam capture images by using a complementary metal oxide silicon sensor, whereas EndoCapsule and the OMOM capsule use a charge-coupled device. ${ }^{5}$ In 2013, the third generation of PillCam SB3 was launched and received FDA clearance. This capsule system has improved image detail and adaptive frame rate technology (two to six frames per second [fps]), leading to increased visualization of the small bowel and improved efficiency. ${ }^{6}$ The CapsoCam has four cameras that provide a $360^{\circ}$ field of view, 12 to $20 \mathrm{fps}$, and 15 hours battery life. The CapsoCam camera takes images at a rate of $5 \mathrm{fps}$ for the first 2 hours and thereafter at a rate of $3 \mathrm{fps}$, resulting in 12 and $20 \mathrm{fps}$, respectively. Smart Motion Sense Technology also enables the capsule to activate its cameras only during capsule motion. ${ }^{7}$

To improve the specificity of small-bowel CE findings, fecal calprotectin (FC) is considered a noninvasive, "gold standard" marker of GI inflammation. An FC level of $>100 \mu \mathrm{g} / \mathrm{g}$ is a good predictor of positive small-bowel CE findings, and FC
$>200 \mu \mathrm{g} / \mathrm{g}$ is associated with higher small-bowel CE yield (65\%) and confirmed $\mathrm{CD}$ in $50 \%$ of patients. ${ }^{8}$

\section{Colon and esophagus capsule endoscopy}

Colorectal cancer (CRC) screening programs in high-risk populations were reported to result in a $90 \%$ decrease in CRC incidence. However, no more than $25 \%$ compliance has been achieved in screening programs. ${ }^{9}$ Colon CE could be a good alternative in patients refusing conventional colonoscopy or when conventional colonoscopy is inappropriate or not possible. In a series of 328 consecutive cases, the rate of complete colon visualization before the end of the lifetime of the battery was $92.8 \% .^{10}$ In two prospective studies with the newer colon capsule endoscope PillCam COLON 2 (Given Imaging), the sensitivity reached $84 \%$ and $89 \%$ for detecting polyps $>6$ mm. ${ }^{11,12}$ Although colon CE showed similar detection capabilities when compared with conventional colonoscopy in some studies, conventional colonoscopy remains more accurate than colon $\mathrm{CE}$, and allows the simultaneous removal of polyps. The colon capsule has a potential future for CRC screening; however, more data are needed to answer many pending uncertainties such as the best preparation method, best scoring method (CECDAI [CE CD Activity Index] or Niv score), best booster, and suitable indications.

In 2004, the PillCam ESO (Given Imaging), which is also capable of studying the esophagus, was developed and approved by the FDA. It has two lenses at both ends and takes 18 images per second over approximately 30 minutes to maximize visualization. Although CE can be used for detecting esophageal diseases such as esophageal varices, Barrett's esophagus, and esophageal cancer, conventional upper GI endoscopy is still the gold standard. 


\section{Improvement of detectability during small-bowel investigation}

\section{In vitro chromoendoscopy}

The Fujinon intelligent chromoendoscopy (FICE) system is a new virtual chromoendoscopy technique that processes reflected photons to reconstruct virtual images with a choice of different wavelengths by using computerized spectral estimation technology. The addition of the FICE technology to small-bowel CE may improve diagnostic yield. However, there is some controversy concerning its effectiveness. In a study by Gupta et al., ${ }^{13}$ FICE-assisted small-bowel CE analysis was no better than analysis with white light for the diagnosis and characterization of significant lesions in the evaluation of OGIB. Matsumura et al. ${ }^{14}$ found that although there was no improvement in diagnostic yield, FICE detected a significantly higher number of small-bowel lesions per examination than did conventional imaging (2.5 \pm 2.1 and $1.8 \pm 1.7$, respectively). Krystallis et al. ${ }^{15}$ compared FICE and white light in a total of 167 images. FICE was ineffective in improving endoscopic images except in the blue mode. Blue filter provided image improvement in $83 \%$ of images when compared with white light. Imagawa et al. ${ }^{16}$ suggested the usefulness of FICE for visualizing small-bowel lesions such as angioectasia, erosions, ulcerations, and various tumors, in a retrospective study. However, in a prospective study, FICE improved the detectability of only angioectasia. ${ }^{16}$

Efficient microcancer detection in the small intestine can be realized with infrared fluorescence endoscopy. Infrared fluorescence levels emitted by the fluorophore indocyanine green (ICG) at different concentrations are able to discriminate low concentrations of ICG in early cancer in the small intestine. ${ }^{17}$

\section{Three-dimensional reconstruction}

In recent years, research has been carried out to produce a three-dimensional (3D) reconstruction of the GI tract; 3D imaging in $\mathrm{CE}$ is not currently feasible because of hardware limitations (i.e., packaging and size constraints, and power consumption). As an alternative method, a software-based approach (shape from shading) that enables $3 \mathrm{D}$ reconstruc- tion from monocular 2D images has become available. ${ }^{18} \mathrm{Kou}-$ laouzidis et al. ${ }^{19}$ determined enhanced visualization for $56 \%$ of vascular and $<10 \%$ of protruding structures $(p=0.007$ and $p=0.008$, respectively). Rondonotti et al. ${ }^{20}$ showed that the adjunction of $3 \mathrm{D}$ reconstructions to their standard 2D counterparts does not enhance the performance of expert small-bowel CE readers ( $p=0.245$ ), although it significantly improves the performance of novices in differentiating masses from bulges $(p=0.045)$.

\section{LIMITATIONS OF CURRENT CAPSULE ENDOSCOPY}

CE has many advantages, but also has several drawbacks (Table 2). Fortunately, many promising solutions have been proposed to resolve these problems (Table 3).

\section{Incomplete small-bowel examination}

\section{Air insufflation}

During its transition along the GI tract, the capsule endoscope is limited by air inflation in exposing the entire mucosa. Several groups have developed a novel device to achieve untethered controlled carbon dioxide $\left(\mathrm{CO}_{2}\right)$ insufflation suitable for $\mathrm{CE}$. This device shows the feasibility of controlled inflation to facilitate visualization. ${ }^{21,22}$

\section{Retention or delayed transition}

The most common causes of incomplete examinations are delayed gastric emptying and prolonged small-bowel transit. These result in the exhaustion of the battery before the capsule reaches the cecum. Administration of water or intravenous metoclopramide could be used in an effort to overcome this problem; however, it is necessary to use this method carefully in patients with dysmotility, as rapid transit time may diminish the diagnostic yield. Use of the external real-time viewer to check the progress of the capsule significantly improved the completion rate ( $86 \%$ vs. $66 \%, p=0.002)$ and the rate of positive findings ( $80 \%$ vs. $67 \%, p=0.04$ ) compared with the

Table 2. Advantages and Disadvantages of Capsule Endoscopy

\begin{tabular}{ll}
\hline Advantages & Disadvantages \\
\hline Convenience & Incomplete small-bowel examination \\
\hline No need for sedation & Uncontrolled air insufflation \\
\hline \multicolumn{1}{c}{ Simple examination for patient } & Retention or delayed transition \\
& Limited battery life \\
\hline Less invasiveness & Impossible to maneuver \\
\hline High diagnostic yield comparable to other imaging modality & No therapeutic or biopsy capability \\
\hline
\end{tabular}


Table 3. New Devices and Future Development

\begin{tabular}{|ll}
\hline Disadvantage & Promising solution \\
\hline Incomplete small bowel examination & \\
\hline Low quality image & FICE, IRFE, 3D reconstruction \\
\hline Uncontrolled air insufflation & Untethered controlled $\mathrm{CO}_{2}$ insufflation \\
\hline Retention or delayed transition & External real-time image viewer \\
\hline Limited battery life & Frame rate modulation \\
& Video compression \\
\hline Location & Impulse Radio-Ultra-Wideband \\
& Software using 3D triangulation \\
\hline Impossibility of maneuver & Capsule-odometer \\
\hline Therapeutic or biopsy capability & Magnetic navigation system \\
& Mobile robotic platform \\
\hline Delayed time of the interpretation & Tagging, biopsy and therapeutic equipment \\
& Targeted drug delivery \\
\hline
\end{tabular}

FICE, Fujinon Intelligent Color Enhancement; IRFE, infrared fluorescence endoscopy; 3D, three-dimensional.
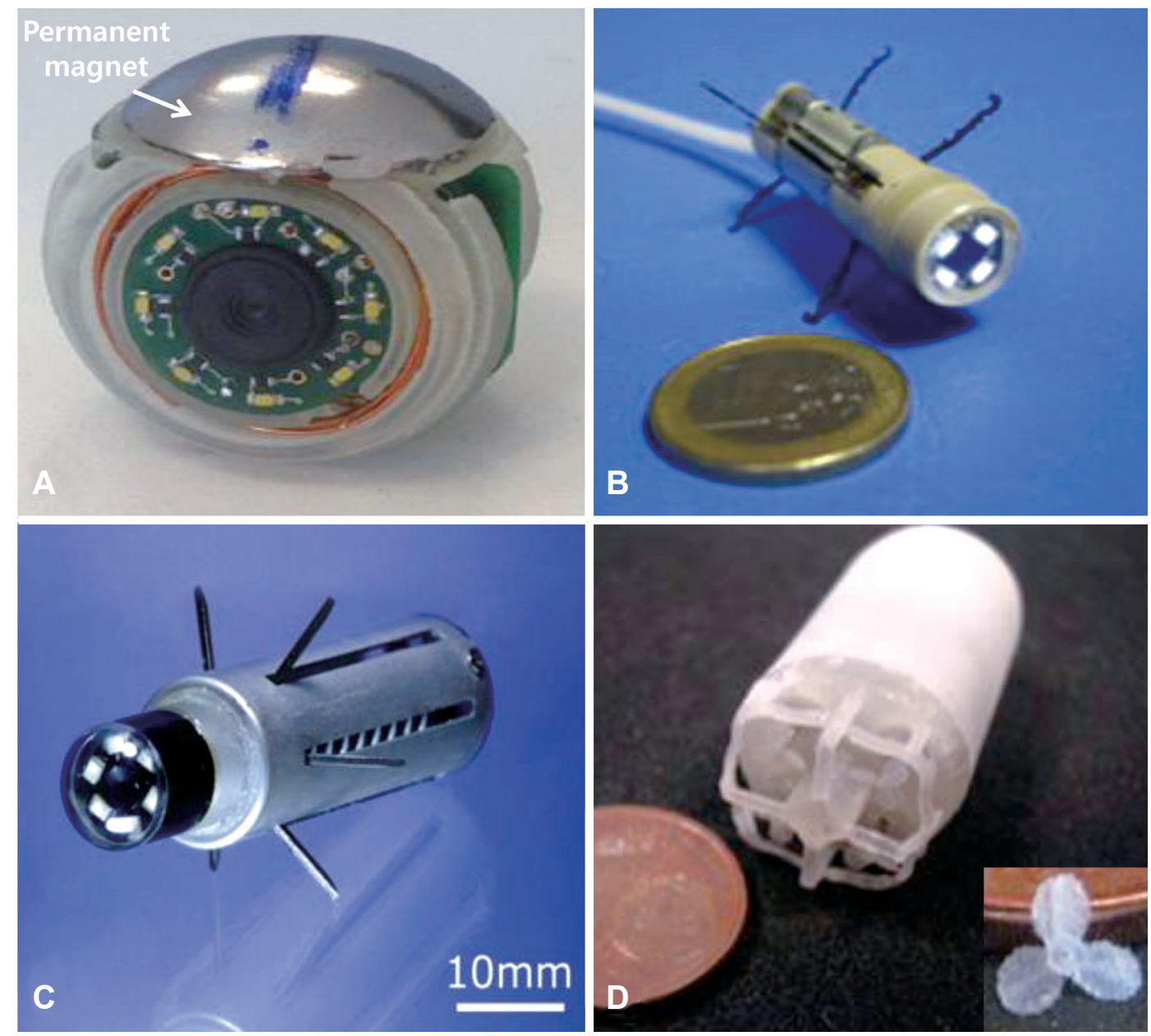

Fig. 1. New capsule endoscope proposed for self- or external ordinary positioning or propulsion. (A) A capsule that emits magnetic force. (B) A capsule with legs for mucosal ambulation. (C) A capsule that involves use of a paddling stroke. (D) A capsule with four propellers. Permissions for all pictures were obtained. (A) Adapted from Lucarini et al. ${ }^{28}(\mathrm{~B}, \mathrm{C})$ Adapted from Quirini et al. ${ }^{29}$ and Kim et al., ${ }^{30}$ with permission from Elsevier, respectively. (D) Adapted from Tortora et al., ${ }^{31}$ with permission from Taylor \& Francis. 
nonviewer group. ${ }^{23}$ In addition, retention can be detected by localization using a capsule emitting a magnetic field or electromagnetic waves. Olympus Medical Systems Corporation has developed new software with $3 \mathrm{D}$ triangulation. ${ }^{24}$ In a study on this method, the average total spatial error with attenuation was $13.26 \mathrm{~cm}^{3}$.

\section{Low battery life}

The capsule battery life is usually 8 to 15 hours. Size reduction and battery life extension have become important challenges, as novel capsule endoscopes and accessory tools have been developed to improve diagnostic yield and perform therapeutic work. Frame rate modulation decreases the frame rate outside of the targeted area and saves battery power, yielding a longer operating time. Consequently, completion rate and diagnostic yield has increased significantly. ${ }^{25}$ Consumption can also be reduced by video compression and transmission technology such as compressed sensing theory and impulse radio ultra-wideband. ${ }^{26}$ One group has proposed primary magnetic coils in a power-generating device outside of the body to send power to a capsule within the body to save space. ${ }^{27}$

\section{Controlled locomotion and positioning of the cap- sule endoscope}

Currently, the movement of the capsule is absolutely dependent on gravitational and peristaltic force. Thus, many methods have been proposed for self- or external ordinary positioning or propulsion (Fig. 1). ${ }^{28-31}$ Although there are differences among capsule models, they are classified into three types: magnetic force, motion of leg or paddle, and propeller. The magnetic-enabled PillCam and magnetic upper GI Olympus capsules have been devised for this purpose. The magnetic-enabled PillCam was based on the Niobe magnetic navigation system (Stereotaxis, St Louis, MO, USA). The core of this system consists of focused-field permanent magnets, made of a neodymium-iron-boron compound. These large magnets are mounted on automatically operated arms to be easily arranged and oriented on either side. In an in vivo experiment, the capsules showed an accuracy of $1^{\circ}$ and a localization error of $1 \mathrm{~mm} .{ }^{32}$ In another study, the camera could be rotated in steps of $1.8^{\circ}$. Full $360^{\circ}$ visualization was possible in the stomach but only a $45^{\circ}$ visual field was possible in the colon. ${ }^{33}$ In 52 human subjects, a feasibility study on a magnetically guided capsule from Olympus demonstrated that visualization of the antrum, body, fundus, and cardia were at $98 \%, 96 \%, 73 \%$, and $75 \%$, respectively, and the feasibility of gastric examination was shown. ${ }^{34}$

Endoscopic devices with flexible legs for ambulation have also been proposed. Legged locomotion mimicking inchworm motion has several advantages, including better adaptability to different geometries of the GI tract, higher velocity, and simplified adhesion induced by friction between the device and the tissue. ${ }^{35} \mathrm{~A}$ new prototype with eight legs has been devised. The two leg sets (each leg set consists of four legs) open independently in opposite directions; the rear legs are useful for propulsion, whereas the front legs are useful for stopping. In in vitro tests, the eight-leg capsule showed a speed of $6 \mathrm{~cm}$ per minute, and capabilities of backward and vertical locomotion. ${ }^{36}$ A paddling-based locomotion mechanism enhanced CE by using a paddling stroke. It provided fast locomotion speed and long travel distances. In one study, the mean velocity was $37.5 \mathrm{~cm}$ per minute in the extracted porcine colon and $17 \mathrm{~cm}$ per minute in the colon of a living pig. ${ }^{37}$ Another study reported that $\mathrm{CE}$ with feedback controlled paddling had higher locomotion speed, showing an increase of 58\% compared with the previous control method based on a given timer value. ${ }^{38} \mathrm{~A}$ capsule endoscope with an integrated propeller has been developed, and recently, a novel capsule endoscope with four propellers has allowed for a reliable 3D locomotion if the capsule has neutral buoyancy. The number of blades per propeller usually varies from one to five, although three-blade propellers are commonly used. ${ }^{39}$ However, this type was useful only for situations in which a large amount of liquid was present. Therefore, liquid intake is required for filling the GI cavity.

For better locomotion and steering, some groups have suggested a hybrid of the magnetic system and self-propelled capsule endoscope, as well as of the magnetic system and legged locomotion. ${ }^{40,41}$ However, these methods require further development concerning the need for bulky and complex equipment for generating the magnetic field, and concerning accuracy and stabilization levels, which remain relatively low.

\section{Beyond detecting lesions (ongoing projects)}

A tagging module can mark the precise location of a target lesion for future surgical or wired endoscopic therapy. ${ }^{42}$ One prototype microbiopsy module consists of a trigger with a paraffin block, a rotating tissue-cutting razor with torsion spring, and a controller. It is constructed to operate sequentially so that the tissue sampling, sealing, and fixing are performed in one operation. ${ }^{43}$ Another form with a microactuator has been designed to perform microbiopsy. A microspike was incorporated into the capsule endoscope to obtain biopsy specimens. Experimental tests demonstrated that the developed microactuator with microspike successfully extracted tissue samples from a pig's small intestines. ${ }^{44}$ One study reported that a magnetically maneuvered capsule with a nitinol clip-releasing mechanism successfully clipped an iatrogenic bleeding lesion in a pig model. ${ }^{45}$ Two new capsules, Intellisite 


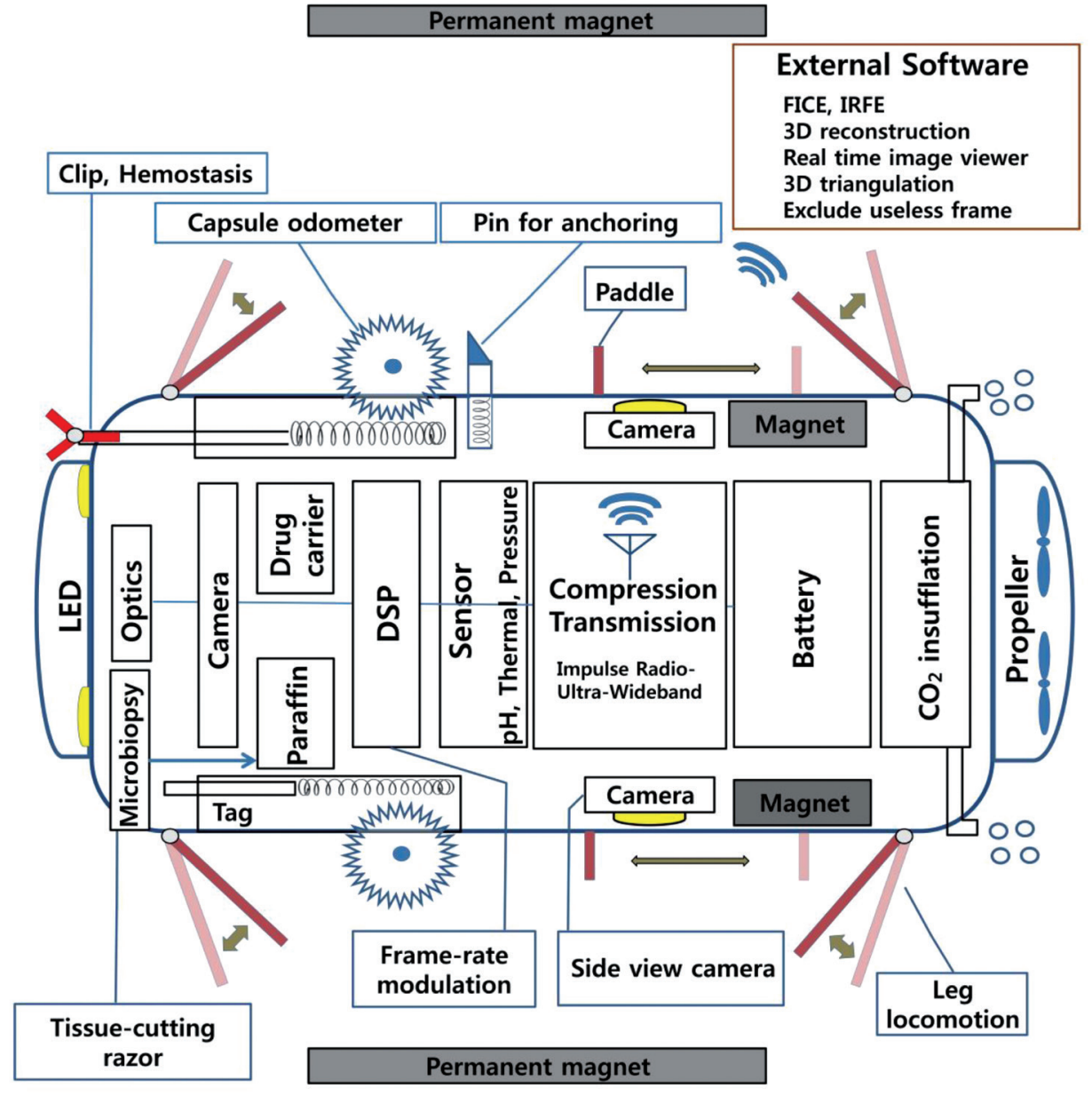

Fig. 2. Schematic illustration of future imaginary capsule endoscopy based on current research. This illustration was made by the present author, Won Gun Kwack. FICE, Fujinon intelligent chromoendoscopy; IRFE, infrared fluorescence endoscopy; 3D, three-dimensional; LED, light-emitting diode; DSP, digital signal processing.
(Innovative Devices, Raleigh, NC, USA) and Enterion (Phaeton Research, Nottingham, UK), have been developed for the collection of absorption data in the GI tract and can be used in the future for drug delivery. ${ }^{46}$ Nonvideo capsules that can deliver drugs with a $\mathrm{pH}$-activated or temperature-activated release mechanism have also been evaluated. Wood et al. reported that a capsule endoscope prototype with $\mathrm{pH}$, temperature, and pressure sensing, and a pin for anchoring with a holding mechanism, demonstrates the feasibility for targeted drug delivery. ${ }^{47}$

The NEMO (nano-based CE with molecular imaging and optical biopsy) project is developing a new capsule that combines optical and maneuvering technologies, biosensing, and nanotechnologies to enhance the diagnostic and therapeutic potential of CE. The VECTOR (versatile endoscopic capsule for GI tumor recognition and therapy) project is in the process of developing a minirobot for the screening and surveillance of GI cancer, and for magnetic and legged motion, drug delivery, and tissue sampling. ${ }^{3,48}$ A prototype coagulation capsule that employs an exothermic chemical reaction to generate heat through the interaction of calcium oxide and water has been tested. ${ }^{49}$ This may be potentially useful for hemostasis through thermal coagulation. However, improvements in capsule maneuvering capabilities are necessary before these capsules can be further developed.

\section{CONCLUSIONS}

$\mathrm{CE}$ has evolved very rapidly to become an important tool for the visualization of the gut mucosa. Small-bowel CE is recommended as the first-line investigation technique in patients with OGIB and seems sufficiently accurate as an alternative tool in other small-bowel diseases such as CD, small tumors, celiac disease, unexplained abdominal pain, and/or diarrhea. For complete and perfect small-bowel investigation, several technical limitations must be addressed. Fortunately, many methods have been proposed and are in development, such as enhanced image modality, controlled air insufflation, decreased battery consumption, and several therapeutic and biopsy tools (Fig. 2). With technological developments, advanced CE could become the standard method of endoscopy 
for many GI diseases in the future.

\section{Conflicts of Interest}

The authors have no financial conflicts of interest.

\section{REFERENCES}

1. Rondonotti E, Soncini M, Girelli C, et al. Small bowel capsule endoscopy in clinical practice: a multicenter 7-year survey. Eur J Gastroenterol Hepatol 2010;22:1380-1386.

2. Koulaouzidis A, Rondonotti E, Karargyris A. Small-bowel capsule endoscopy: a ten-point contemporary review. World J Gastroenterol 2013;19:3726-3746.

3. Fisher LR, Hasler WL. New vision in video capsule endoscopy: current status and future directions. Nat Rev Gastroenterol Hepatol 2012;9:392405.

4. Eliakim R. Video capsule endoscopy of the small bowel. Curr Opin Gastroenterol 2013;29:133-139.

5. Ladas SD, Triantafyllou K, Spada C, et al. European Society of Gastrointestinal Endoscopy (ESGE): recommendations (2009) on clinical use of video capsule endoscopy to investigate small-bowel, esophageal and colonic diseases. Endoscopy 2010;42:220-227.

6. Bouchard S, Ibrahim M, Van Gossum A. Video capsule endoscopy: perspectives of a revolutionary technique. World J Gastroenterol 2014;20:17330-17344.

7. Van de Bruaene C, De Looze D, Hindryckx P. Small bowel capsule endoscopy: Where are we after almost 15 years of use? World J Gastrointest Endosc 2015;7:13-36.

8. Koulaouzidis A, Douglas S, Rogers MA, Arnott ID, Plevris JN. Fecal calprotectin: a selection tool for small bowel capsule endoscopy in suspected IBD with prior negative bi-directional endoscopy. Scand J Gastroenterol 2011;46:561-566.

9. Winawer SJ, Zauber AG, Ho MN, et al. Prevention of colorectal cancer by colonoscopic polypectomy. The National Polyp Study Workgroup. N Engl J Med 1993;329:1977-1981.

10. Van Gossum A, Munoz-Navas M, Fernandez-Urien I, et al. Capsule endoscopy versus colonoscopy for the detection of polyps and cancer. $\mathrm{N}$ Engl J Med 2009;361:264-270.

11. Spada C, Hassan C, Munoz-Navas M, et al. Second-generation colon capsule endoscopy compared with colonoscopy. Gastrointest Endosc 2011;74:581-589.e1.

12. Eliakim R, Yassin K, Niv Y, et al. Prospective multicenter performance evaluation of the second-generation colon capsule compared with colonoscopy. Endoscopy 2009;41:1026-1031.

13. Gupta T, Ibrahim M, Deviere J, Van Gossum A. Evaluation of Fujinon intelligent chromo endoscopy-assisted capsule endoscopy in patients with obscure gastroenterology bleeding. World J Gastroenterol 2011;17:4590-4595.

14. Matsumura T, Arai M, Sato T, et al. Efficacy of computed image modification of capsule endoscopy in patients with obscure gastrointestinal bleeding. World J Gastrointest Endosc 2012;4:421-428.

15. Krystallis C, Koulaouzidis A, Douglas S, Plevris JN. Chromoendoscopy in small bowel capsule endoscopy: blue mode or Fuji Intelligent Colour Enhancement? Dig Liver Dis 2011;43:953-957.

16. Imagawa $\mathrm{H}$, Oka $\mathrm{S}$, Tanaka $\mathrm{S}$, et al. Improved detectability of small-bowel lesions via capsule endoscopy with computed virtual chromoendoscopy: a pilot study. Scand J Gastroenterol 2011;46:1133-1137.

17. Demosthenous P, Georgiou J. Towards a fluoroscopic cancer screening capsule for the small intestine. Conf Proc IEEE Eng Med Biol Soc 2014;2014:3122-3125.

18. Zhang R, Tsai PS, Cryer JE, Shah M. Shape-from-shading: a survey. IEEE Trans Pattern Anal Mach Intell 1999;21:690-706.
19. Koulaouzidis A, Karargyris A, Rondonotti E, et al. Three-dimensional representation software as image enhancement tool in small-bowel capsule endoscopy: a feasibility study. Dig Liver Dis 2013;45:909-914.

20. Rondonotti E, Koulaouzidis A, Karargyris A, et al. Utility of 3-dimensional image reconstruction in the diagnosis of small-bowel masses in capsule endoscopy (with video). Gastrointest Endosc 2014;80:642-651.

21. Pasricha T, Smith BF, Mitchell VR, et al. Controlled colonic insufflation by a remotely triggered capsule for improved mucosal visualization. Endoscopy 2014;46:614-618.

22. Gorlewicz JL, Battaglia S, Smith BF, et al. Wireless insufflation of the gastrointestinal tract. IEEE Trans Biomed Eng 2013;60:1225-1233.

23. Shiotani A, Honda K, Kawakami M, et al. Use of an external real-time image viewer coupled with prespecified actions enhanced the complete examinations for capsule endoscopy. J Gastroenterol Hepatol 2011;26:1270-1274.

24. Marya N, Karellas A, Foley A, Roychowdhury A, Cave D. Computerized 3-dimensional localization of a video capsule in the abdominal cavity: validation by digital radiography. Gastrointest Endosc 2014;79:669674 .

25. Liao Z, Xu C, Li ZS. Completion rate and diagnostic yield of small-bowel capsule endoscopy: 1 vs. 2 frames per second. Endoscopy 2010;42:360364.

26. Gao Y, Zheng Y, Diao S, et al. Low-power ultrawideband wireless telemetry transceiver for medical sensor applications. IEEE Trans Biomed Eng 2011;58:768-772.

27. Xin W, Yan G, Wang W. Study of a wireless power transmission system for an active capsule endoscope. Int J Med Robot 2010;6:113-122.

28. Lucarini G, Ciuti G, Mura M, Rizzo R, Mensiacci A. A new concept for magnetic capsule colonoscopy based on an electromagnetic system. Int J Adv Robot Syst 2015;12:25.

29. Quirini M, Menciassi A, Scapellato S, et al. Feasibility proof of a legged locomotion capsule for the GI tract. Gastrointest Endosc 2008;67:11531158.

30. Kim HM, Yang S, Kim J, et al. Active locomotion of a paddling-based capsule endoscope in an in vitro and in vivo experiment (with videos). Gastrointest Endosc 2010;72:381-387.

31. Tortora G, Valdastri P, Susilo E, et al. Propeller-based wireless device for active capsular endoscopy in the gastric district. Minim Invasive Ther Allied Technol 2009;18:280-290.

32. Carpi F, Pappone C. Stereotaxis Niobe magnetic navigation system for endocardial catheter ablation and gastrointestinal capsule endoscopy. Expert Rev Med Devices 2009;6:487-498.

33. Valdastri P, Quaglia C, Buselli E, et al. A magnetic internal mechanism for precise orientation of the camera in wireless endoluminal applications. Endoscopy 2010;42:481-486.

34. Rey JF, Ogata H, Hosoe N, et al. Feasibility of stomach exploration with a guided capsule endoscope. Endoscopy 2010;42:541-545.

35. Menciassi A, Stefanini C, Gorini S, et al. Locomotion of a legged capsule in the gastrointestinal tract: theoretical study and preliminary technological results. Conf Proc IEEE Eng Med Biol Soc 2004;4:2767-2770.

36. Quirini M, Scapellato S, Valdastri P, Menciassi A, Dario P. An approach to capsular endoscopy with active motion. Conf Proc IEEE Eng Med Biol Soc 2007;2007:2827-2830.

37. Kim HM, Yang S, Kim J, et al. Active locomotion of a paddling-based capsule endoscope in an in vitro and in vivo experiment (with videos). Gastrointest Endosc 2010;72:381-387.

38. Yang S, Park K, Kim J, Kim TS, Cho IJ, Yoon ES. Autonomous locomotion of capsule endoscope in gastrointestinal tract. Conf Proc IEEE Eng Med Biol Soc 2011;2011:6659-6663.

39. Tortora G, Valdastri P, Susilo E, et al. Propeller-based wireless device for active capsular endoscopy in the gastric district. Minim Invasive Ther Allied Technol 2009;18:280-290.

40. Gao M, Hu C, Chen Z, Zhang H, Liu S. Design and fabrication of a magnetic propulsion system for self-propelled capsule endoscope. IEEE Trans Biomed Eng 2010;57:2891-2902. 
41. Simi M, Valdastri P, Quaglia C, Menciassi A, Dario P. Design, fabrication, and testing of a capsule with hybrid locomotion for gastrointestinal tract exploration. Mechatron IEEE/ASME Trans 2010;15:170-180.

42. Chandrappan J, Ruiqi L, Su N, Qiang TS, Vaidyanathan K. Tagging module for lesion localization in capsule endoscopy. Conf Proc IEEE Eng Med Biol Soc 2010;2010:1890-1893.

43. Kong KC, Cha J, Jeon D, Cho DI. A rotational micro biopsy device for the capsule endoscope. In: Proceedings of the Intelligent Robots and Systems, 2005 (IROS 2005) 2005 IEEE/RSJ International Conference; 2005 Aug 2-6; Edmont, AB, Canada. IEEE/RSJ International Conference: IEEE; 2005. p. 1839-1843.

44. Park S, Koo KI, Bang SM, Park JY, Song SY, Cho DD. A novel microactuator for microbiopsy in capsular endoscopes. J Micromech Microeng
2008;18:025032.

45. Valdastri P, Quaglia C, Susilo E, et al. Wireless therapeutic endoscopic capsule: in vivo experiment. Endoscopy 2008;40:979-982.

46. Wilding II, Hirst P, Connor A. Development of a new engineering-based capsule for human drug absorption studies. Pharm Sci Technolo Today 2000;3:385-392.

47. Woods SP, Constandinou TG. Wireless capsule endoscope for targeted drug delivery: mechanics and design considerations. IEEE Trans Biomed Eng 2013;60:945-953.

48. Schostek S, Schurr MO. European research on wireless endoscopy: the VECTOR project. Stud Health Technol Inform 2013;189:193-199.

49. Swain P. The future of wireless capsule endoscopy. World J Gastroenterol 2008;14:4142-4145. 